\title{
La proteinuria elevada es un factor de riesgo independiente de infarto de miocardio, progresión de la nefropatía y mortalidad
}

Elevated proteinuria is an independent risk factor for myocardial infarction, nephropathy progression, and death

Hemmelgarn y col. JAMA 2010;303(5):423-9.

\section{Objetivo}

Determinar la asociación entre el nivel de filtrado glomerular, la proteinuria y los eventos clínicos adversos.

\section{Diseño, lugar y pacientes}

Estudio de cohortes comunitario* realizado en Alberta, Canadá. Participaron 920.985 adultos mayores de 18 años con al menos un dosaje ambulatorio de creatininemia durante el periodo de mayo de 2002 a diciembre de 2006. Fueron excluidos los pacientes con enfermedad renal crónica terminal.

\section{Evaluación de los factores pronósticos}

Las principales variables examinadas fueron: el filtrado glomerular calculado por el método MDRD', la proteinuria medida en muestras aisladas de orina mediante tiras reactivas $\mathrm{o}$, en una fracción de la población, mediante el cociente albumina/creatinina (ACR).

\section{Medición de resultados principales}

El resultado primario* fue la mortalidad general, y los secundarios*, el infarto de miocardio y la progresión de la nefropatía.

\section{Resultados Principales}

Durante una mediana de 35 meses de seguimiento fallecieron unos 27.959 participantes (3\%). La tasa de mortalidad general ajustada*, así como el resto de los resultados secundarios, fue proporcionalmente mayor en aquellas personas con filtrado glomerular reducido o proteinuria elevada (ver tabla 1). El riesgo absoluto de eventos en el subgrupo de pacientes (102.701) en que se determino el nivel de proteinuria mediante ACR fue mayor (datos no mostrados en la tabla).

Tabla 1: tasa ajustada ${ }^{\delta}$ de eventos clínicos, por cada 1000 personas año, según el nivel de filtrado glomerular y proteinuria medida por tiras reactivas.

\begin{tabular}{|c|c|c|c|c|c|c|c|c|c|c|c|c|}
\hline & \multicolumn{12}{|c|}{ Nivel de Proteinuria y eventos clínicos } \\
\hline & \multicolumn{3}{|c|}{ Mortalidad general } & \multicolumn{3}{|c|}{ Infarto de miocardio } & \multicolumn{3}{|c|}{ Enfermedad renal Terminal } & \multicolumn{3}{|c|}{ Progresión de la nefropatia $^{\varepsilon}$} \\
\hline & Normal $^{a}$ & Leve $^{\beta}$ & Severa $^{\gamma}$ & Normal & Leve & Severa & Normal & Leve & Severa & Normal & Leve & Severa \\
\hline$\geq 60$ & $\begin{array}{c}2,7 \\
(2,6 \text { a } 2,8)\end{array}$ & \begin{tabular}{c|}
5,8 \\
$(5,5 \mathrm{a} 6,0)$ \\
\end{tabular} & $\begin{array}{c}7,2 \\
(6,6 \text { a } 7,8)\end{array}$ & $\begin{array}{c}0,9 \\
(0,9 \text { a } 1,0)\end{array}$ & $\begin{array}{c}1,3 \\
(1,2 \mathrm{a} 1,5)\end{array}$ & $\begin{array}{c}1,6 \\
(1,3 \text { a } 2,0)\end{array}$ & $\begin{array}{c}0,03 \\
(0,02 \text { a } 0,03)\end{array}$ & $\begin{array}{c}0,05 \\
(0,03 \text { a } 0,09)\end{array}$ & $\begin{array}{c}1,0 \\
(0,7 \text { a } 1,4)\end{array}$ & $\begin{array}{c}0,6 \\
(0,5 \mathrm{a} 0,6)\end{array}$ & $\begin{array}{c}1,6 \\
(1,4 \text { a } 1,9)\end{array}$ & $\begin{array}{c}5,9 \\
(5,0 \text { a } 7,0)\end{array}$ \\
\hline 45 & $\begin{array}{c}2,9 \\
(2,7 \text { a } 3,0)\end{array}$ & \begin{tabular}{|c|}
5,2 \\
$(4,9$ a 5,5$)$ \\
\end{tabular} & $\begin{array}{c}7,2 \\
(6,5 \text { a } 7,8)\end{array}$ & $\begin{array}{c}1,2 \\
(1,1 \mathrm{a} 1,2)\end{array}$ & $\begin{array}{c}1,3 \\
(1,1 \text { a } 1,5)\end{array}$ & $\begin{array}{c}1,8 \\
(1,4 \text { a } 2,3)\end{array}$ & $\begin{array}{c}0,2 \\
(0,1 \mathrm{a} 0,2)\end{array}$ & $\begin{array}{c}0,7 \\
(0,5 \text { a } 1,2)\end{array}$ & $\begin{array}{c}4,3 \\
(3,1 \text { a } 6,1)\end{array}$ & $\begin{array}{c}0,9 \\
(0,8 \text { a } 1,0)\end{array}$ & \begin{tabular}{c|}
2,1 \\
$(1,7$ a 2,5$)$
\end{tabular} & $\begin{array}{c}10,0 \\
(8,3 \text { a } 11,9)\end{array}$ \\
\hline 30 a 44,9 & $\begin{array}{c}4,0 \\
(3,7 \mathrm{a} 4,2) \\
\end{array}$ & \begin{tabular}{|c|}
5,8 \\
$(5,4 \mathrm{a} 6,2)$ \\
\end{tabular} & $\begin{array}{c}7,5 \\
(6,8 \text { a } 8,2) \\
\end{array}$ & $\begin{array}{c}1,4 \\
(1,3 \mathrm{a} 1,6) \\
\end{array}$ & $\begin{array}{c}1,5 \\
(1,2 \mathrm{a} 1,8) \\
\end{array}$ & $\begin{array}{c}2,1 \\
(1,6 \text { a } 2,7) \\
\end{array}$ & $\begin{array}{c}1,3 \\
(0,9 \text { a } 1,8) \\
\end{array}$ & $\begin{array}{c}4,2 \\
(3,0 \mathrm{a} 6,0) \\
\end{array}$ & $\begin{array}{c}16,1 \\
(12,5 \text { a } 20,7) \\
\end{array}$ & $\begin{array}{c}2,0 \\
(1,7 \text { a } 2,4) \\
\end{array}$ & \begin{tabular}{|c|}
4,7 \\
$(3,9$ a 5,7$)$ \\
\end{tabular} & $\begin{array}{c}12,8 \\
(10,7 \text { a } 15,3) \\
\end{array}$ \\
\hline 5 a 29,9 & $\begin{array}{c}6,7 \\
\text { (6,2 a 7,3) } \\
\end{array}$ & $\begin{array}{c}9,1 \\
(8,2 \text { a } 10,0) \\
\end{array}$ & $\begin{array}{c}10,4 \\
(9,3 \mathrm{a} 11,6) \\
\end{array}$ & $\begin{array}{c}2,1 \\
(1,6 \mathrm{a} 2,6) \\
\end{array}$ & $\begin{array}{c}2,2 \\
(1,6 \text { a } 2,9) \\
\end{array}$ & $\begin{array}{c}3,3 \\
(2,5 \mathrm{a} 4,3) \\
\end{array}$ & $\begin{array}{c}12,7 \\
(9,3 \text { a } 17,3) \\
\end{array}$ & $\begin{array}{c}25,2 \\
\text { (18,9 a 33,4) }\end{array}$ & $\begin{array}{c}65,9 \\
(52,3 \text { a } 82,9)\end{array}$ & $\begin{array}{c}4,5 \\
(3,5 a \text { 5,9) }\end{array}$ & $\begin{array}{c}10,5 \\
(8,3 \text { a } 13,3) \\
\end{array}$ & $\begin{array}{c}24,7 \\
(20,7 \text { a 29,6) } \\
\end{array}$ \\
\hline
\end{tabular}

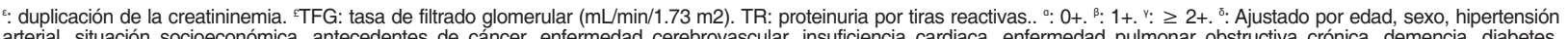
arterial, situación socioeconómica, antecedentes de cancer, enfermedad cerebrovascular, insuficiencia cardiaca, enfermedad pulmonar obstructiva crónica, demencia, diabetes,
HIV/SIDA, tumor metastático sólido, infarto de miocardio, hepatopatia, enfermedad ulcerosa péptica, enfermedad vascular periférica, enfermedad renal y enfermedad reumática.

\section{Conclusión}

La proteinuria elevada es un factor de riesgo independiente de mortalidad general, infarto de miocardio, y progresión de la nefropatía crónica.
Palabras claves: proteinuria, insuficiencia renal crónica, mortalidad, infarto de miocardio. Keywords: proteinuria, chronic renal insufficiency, myocardial infarction. Fuentes de financiamiento: Alberta Heritage Foundation for Medical Reaserch, Canadian Institutes of Health Research, Alberta Health and Wellness, University of Alberta y Calgary, KRESCENT y AHFMR fellowships.

\section{Comentario}

Estudios multicéntricos han demostrado que la mera presencia de microalbuminuria constituye un factor de riesgo cardiovascular independiente en la población general, aún en personas sin hipertensión o diabetes mellitus (PREVEND, HUNT y EPICaNorfolk) $)^{1,2,3}$. La correlación observada entre la presencia de proteinuria y el riesgo cardiovascular centra su fisiopatología en la disfunción endotelial generalizada que aquélla refleja y que a su vez preludia el desarrollo acelerado de ateroesclerosis. Asimismo, el estudio PREVEND, demostró que el tratamiento con IECA (fosinopril) en individuos con microalbuminuria como único factor de riesgo logró una disminución de la misma, asociándose este efecto con una correlativa disminución del riesgo cardiovascular ${ }^{3,4}$. Además, hay evidencia de que el tratamiento temprano de la proteinuria retrasa la progresión hacia enfermedad renal terminal ${ }^{4,5}$. Sin embargo, es importante destacar que a pesar de la evidencia antes mencionada, e incluso ratificada por el presente estudio, la actual estadificación de la nefropatía crónica sólo tiene en cuenta el valor del filtrado glomerular del paciente, y no justamente la magnitud de su proteinuria.

\section{Conclusiones del comentador}

Resulta entonces relevante que el médico tenga en cuenta la presencia de proteinuria a fin de lograr una adecuada ponderación y tratamiento (IECA, bloqueantes del receptor de angiotensina II, espironolactona, inhibidores de la renina) de estos pacientes que ostentan un riesgo incrementado de mortalidad y progresión de deterioro renal.

Carlos G. Musso [ Servicio de Nefrología del Hospital Italiano de Buenos Aires. carlos.musso@ hospitalitaliano.org.arjose.alfie@ hospitalitaliano.org.ar ]

Ver glosario Recibido el 29/04/2010 y aceptado el 05/05/2010 Musso C. La proteinuria elevada es un factor de riesgo independiente de infarto de miocardio, progresión de la nefropatía y mortalidad. Evid. Actual. Práct. Ambul; 13(3):92, Jul-Set 2010. Comentado de: Hemmelgarn B y col. Relation between kidney function, proteinuria, and adverse outcomes. JAMA 2010;303(5):423-9. PMID: 20124537.

\section{Referencias}

1. Diercks GF, y col. Microalbuminuria is independently associated with ischaemic electrocardiographic abnormalities in a large non-diabetic population. The PREVEND (Prevention of REnal and Vascular ENdstage Disease) study. Eur Heart J. 2000 Dec;21(23):1922-7

2. Romundstad S, y col. Microalbuminuria and all-cause mortality in 2,089 apparently healthy individuals: a 4.4-year follow-up study. The Nord-Trondelag Health Study (HUNT). NorwayAm J Kidney Dis 2003;42/3:466-473

3. Yuyun MF, y col. Microalbuminuria independently predicts all-cause and cardiovascular mortality in a British population: the European Prospective Investigation into Cancer in Norfolk (EPIC-Norfolk) population study. Int J Epidemiol 2004;33/1:189-198

4. Renal Pathophysiology, Rennke H, Denker B, Philadelphia, Lippincott Williams \& Wilkins, 2007.

5. Glassock R. Symptomatic therapy. En: Treatment of primary glomerulonephritis. Ponticelli C, Glassock R (Eds), Oxford, Oxford University Press, $2009: 1 a 46$ 